\title{
High rises and earth subsidence
}

Jess Lan Ouyang ${ }^{1}$, Min Fu², Jingli Peng ${ }^{1}$, Man Tang ${ }^{1}$, Qiuyun Liu ${ }^{1, *}$

${ }^{1}$ School of Life Sciences, Sun Yat-Sen University, Guangzhou 510275, China.

${ }^{2}$ Deyang Health Committee, Sichuan, China

*Correspondence author: Qiuyun Liu, School of Life Sciences, Sun Yat-sen University, Guangzhou 510275, China.

E-mail address: liuqiuyunsysu@163.com (Q. Liu) 


\begin{abstract}
Skyscrapers are synonymous with mega cities throughout the world, accompanied with which is earth subsidence. High rises are larger in inertia, and decelerate and accelerate at slower speeds than smaller buildings. The slower deceleration can lead to drift along the direction of Earth's self-rotation and revolution, resulting in the appearance of minute cracks to the west of the skyscrapers and thus earth subsidence.
\end{abstract}

Keywords

Skyscrapers; earth subsidence; decelerate; drift 
Skyscrapers are the landmarks of an industrialized society. They keep shaping the skylines of modern cities. Coming with that is earth subsidence. In order to explore the underling mechanism, we need to turn to the emerging theory of seismic activities (1-5). Large mass such as the Tibetan Plateau possesses large inertia, and is subjected to the influence of solar and lunar gravitational pulls. The slower deceleration of the Tibetan Plateau results in the northeastward drift since the Earth self-rotates and orbits around the Sun.

High rises are larger in inertia, and decelerate and accelerate more slowly than smaller buildings. The slower deceleration can lead to drift along the directions of Earth's self-rotation as well as revolution, resulting in the appearance of minute cracks to the west of the skyscrapers and thus earth subsidence. To address this problem, high rises with approximately equal heights and equal inertia can be constructed along the direction of drift in a region, as skyscrapers will drift at similar speed and in similar direction to avoid cracks.

\section{Conflict of interest}

None declared.

\section{ACKNOWLEDGMENT}

This work was supported by the Science and Technology Transformation Program of Sun Yat-sen University of China (33000-18843234) and Guangzhou Science and Technology Program (201804010328) to Q. Liu. We thank Yan Shi for editing.

\section{REFERENCES}

1. Lin Y, et al. RE: Mechanism of earthquakes: resemblance of cable-stayed bridges. Science, 2020. https://science.sciencemag.org/content/354/6315/1027/tab-e-letters

2. Yan S, et al. Volcanoes or earthquakes: Wrist wrestling. Science, 2019. http://science.sciencemag.org/content/358/6370/1520/tab-e-letters.

3. Yan S, et al. Earthquakes-an intricate trio dance of gravitation. Science 2018. http://science.sciencemag.org/content/354/6315/1027/tab-e-letters.

4. Ran H, et al. Vortex-induced vibration: The slower deceleration of the west bank leads to the shortening of the Humen Bridge in Guangdong, China. OSF Preprints. 2020. doi:10.31219/osf.io/3t4a6. https://osf.io/3t4a6

5. Lu X, et al. 2020. The revolution of the earth around the sun causes the vortex-induced vibration of south-north trending bridges and impacts seismic activities. OSF Preprints. 2020. https://doi.org/10.31219/osf.io/ycjhz

6. Liao L, et al. Differential inertia of lower and upper parts of a skyscraper drives swaying of a building. OSF Preprints. 2021. https://doi.org/10.31219/osf.io/ztvsk 